\title{
Transcriptome expression analysis of candidate milk genes affecting cheese-related traits in 2 sheep breeds
}

\author{
A. Suárez-Vega, B. Gutiérrez-Gil, and J. J. Arranz ${ }^{1}$ \\ Departamento de Producción Animal, Facultad de Veterinaria, Universidad de León, Campus de Vegazana s/n, León 24071, Spain
}

\begin{abstract}
Because ewe milk is principally used for cheese making, its quality is related to its content of total solids and the way in which milk constituents influence cheese yield and determine the technological and organoleptic characteristics of dairy products. Therefore, an in-depth knowledge of the expression levels of milk genes influencing cheese-related traits is essential. In the present study, the milk transcriptome data set of 2 dairy sheep breeds, Assaf and Spanish Churra, was used to evaluate the expression levels of 77 transcripts related to cheese yield and quality traits. For the comparison between both breeds, we selected the RNA sequencing (RNASeq) data at d 10 of lactation because this is the time point at which within and between breed differences due to lactation length are minimal. The evaluated genes encode major milk proteins (caseins and whey proteins), endogenous proteases, and enzymes related to fatty acid metabolism and citrate content. Through this analysis, we identified the genes predominantly expressed in each of the analyzed pathways that appear to be key genes for traits related to sheep milk cheese. Among the highly expressed genes in both breeds were the genes encoding caseins and whey proteins (CSN2, CSN3, CSN1S1, ENSOARG00000005099/PAEP, $C S N 1 S 2, L A L B A$ ), genes related to lipid metabolism (BTN1A1, XDH, FASN, ADFP, SCD, H-FABP, $A C S S 2)$, and one endogenous protease $(C T S B)$. Moreover, a differential expression analysis between Churra and Assaf sheep allowed us to identify 7 genes that are significantly differentially expressed between the 2 breeds. These genes were mainly linked to endogenous protease activity (CTSL, CTSK, KLK10, KLK6, SERPINE2). Additionally, there were 2 differentially expressed genes coding for an intracellular fatty acid transporter $(F A B P 4)$, an intermediate molecule of the
\end{abstract}

Received February 18, 2016.

Accepted April 11, 2016.

${ }^{1}$ Correesponding author: jjarrs@unileon.es citric acid cycle (SUCNR1), and 2 heat shock proteins (HSPr0, HSPB8) that could be related to high protein production. The differential expression of these genes could have a direct influence on the different phenotypes observed between the 2 analyzed breeds.

Key words: dairy sheep, RNA-Seq, gene expression, cheese traits

\section{INTRODUCTION}

Improving milk quality through genetics is not an easy goal. The concept of milk quality involves several factors (constituent contents, nutritional and functional characteristics, technological and organoleptic properties, food safety), many of which can be under genetic control (Moioli et al., 2007). The dairy sheep industry represents a significant part of the agricultural economies of various countries, particularly in the Mediterranean and Middle East regions (Selvaggi et al., 2014), and it is focused on the elaboration of dairy products, mainly high-quality mature cheeses. Because ewe's milk is principally used for cheese making, its quality is related to milk total solids and the way in which milk constituents influence cheese yield and determine the technological and organoleptic characteristics of dairy products. Therefore, an in-depth knowledge of the expression levels of milk genes influencing cheese-related traits is essential.

Cheese primarily contains the fat and casein from milk. Because ovine milk generally contains higher total solids and major nutrient contents than bovine and caprine milk (Selvaggi et al., 2014), sheep milk results in higher cheese yield than cow or goat milk. Sheep milk also has a higher ratio of $\beta: \alpha_{S^{-}}$casein than cow milk, which makes sheep milk more sensitive to rennet and stimulates faster coagulation (Selvaggi et al., 2014). Moreover, the fat-to-protein ratio of milk influences the texture and the melting properties of cheese (Moioli et al., 2007). However, in addition to the main milk proteins and lipids, minor milk components also influence cheese-related traits, such as citrate content and endogenous proteases. Citrate affects milk-processing 
properties because it alters the coagulation of milk proteins and its fermentation products yield distinct aromatic flavors (Rosenthal, 1991). Endogenous proteases contribute to cheese ripening, producing distinct, sapid amino acids that contribute to the flavors of fermented dairy products (McSweeney, 2004).

In the present study, we used whole-transcriptome sequencing analysis (RNA-Seq) of milk somatic cells (MSC) to evaluate the expression levels of 77 transcripts related to cheese yield and cheese quality traits in 2 different sheep breeds. Milk somatic cells have been shown to be a reliable source to study the expression levels of genes in the mammary gland (Canovas et al., 2014). The RNA-Seq technique provides a unique opportunity to quantify transcripts and identify differential expression in a single experiment (Wang et al., 2009). This technology has recently been used by our research group to provide a dynamic characterization of the milk transcriptome through sheep lactation (Suárez-Vega et al., 2015).

The genes evaluated in the present analysis code for major milk proteins (caseins and whey proteins), endogenous proteases, and enzymes related to fatty acid metabolism and citrate content. The RNA was extracted from MSC of 2 dairy sheep breeds, Assaf and Spanish Churra. These breeds were selected based on their different phenotypes in relation to milk production characteristics and cheese-making properties. Assaf sheep have higher milk yield than Churra sheep (425.46 $\mathrm{L}$ normalized to $150 \mathrm{~d}$ vs. $114.72 \mathrm{~L}$ normalized to 120 d), whereas Churra sheep have a higher protein (5.98\%) and fat $(7.12 \%)$ contents in milk than Assaf sheep (4.03 and $5.39 \%$, respectively) (Othmane et al., 2002; PérezCabal et al., 2013). Therefore, milk from Churra sheep has a higher cheese yield than milk from Assaf sheep [22.50\% in Churra versus 20.00\% in Assaf (Revilla et al., 2009)]. Churra milk also has a better aptitude for the production of mature cheeses than Assaf (LurueñaMartinez et al., 2010b). In addition to quantifying gene expression levels, we performed a differential expression analysis between the 2 analyzed breeds to confirm some of the differences observed and to identify additional milk genes influencing cheese-related traits.

\section{MATERIALS AND METHODS}

\section{Animals, Sampling, and RNA Extraction}

For this study, we used the MSC transcriptome data set from Assaf and Spanish Churra dairy sheep breeds available in the Gene Expression Omnibus database under the accession number GSE74825 and previously described (Suárez-Vega et al., 2015). The source of animals, the sampling process protocol, and the RNA extraction and sequencing processes are described in detail in the Materials and Methods section of SuárezVega et al. (2015). Briefly, RNA was extracted from MSC of 8 healthy sheep, 4 Churra and 4 Assaf ewes, on d 10, 50, 120, and 150 after lambing. Selected ewes belong to the commercial farm of the University of León. These sheep were maintained in freestall housing and fed with the same rations and had no water restrictions. All protocols involving animals were approved by the Animal Welfare Committee of the University of Leon, Spain, following proceedings described in Spanish and EU legislations (Law 32/2007, R.D. 1201/2005, and Council Directive 2010/63/EU). A total of $50 \mathrm{~mL}$ of milk from each ewe was collected $1 \mathrm{~h}$ after $0800 \mathrm{~h}$ routine milking and 10 min after the administration of 5 IU of Oxytocin Facilpart (Syva, León, Spain). The time of milk collection was chosen to maximize the concentration of MSC based on previous studies that indicate that $1 \mathrm{~h}$ after milking is the diurnal time point with the highest concentration of MSC (Gonzalo et al., 1994). Oxytocin was administrated only on sampling days to avoid any effect on milk composition and with the aim of stimulating the mechanical effect of myoepithelial contraction and thus the flattering of the alveolar lumen that causes the release of the residual postmilking milk, which has a higher concentration of exfoliated mammary epithelial cells (Peris et al., 1991).

Somatic cell separation and RNA extraction was performed as described by Suárez-Vega et al. (2015). The integrity of the RNA was assessed using an Agilent 2100 Bioanalyzer device (Agilent Technologies, Santa Clara, CA). The RNA integrity value of the samples ranged between 7.1 and 8.5. Paired-end libraries with fragments of $300 \mathrm{bp}$ were prepared using the True-Seq RNA-Seq sample preparation Kit v2 (Illumina, San Diego, CA). The fragments were sequenced on an Illumina Hi-Seq 2000 sequencer (Fasteris SA, Plan-lesOuates, Switzerland).

\section{Selection of Target Genes}

Target genes included those coding for major milk constituent proteins (caseins and whey proteins) and other genes that were selected based on KEGG pathways and public reviews on 4 aspects potentially related to cheese-related traits. Seventy-seven genes were selected (Supplementary Tables S1 and S2; http:// dx.doi.org/10.3168/jds.2016-11048): 7 genes encoding caseins and whey proteins, 17 genes related to mammary gland lipid metabolism (Bionaz and Loor, 2008), 22 genes related to citrate metabolism (KEGG pathway oas00020 and Cánovas et al., 2013), and 31 genes 
encoding endogenous proteases (Wickramasinghe et al., 2012; Suárez-Vega et al., 2015).

\section{RNA-Seq Analysis}

The read qualities of the RNA-Seq libraries were evaluated using FastQC (http://www.bioinformatics.babraham.ac.uk/projects/fastqc). The reads were mapped using the STAR aligner (Dobin et al., 2013) against the ovine genome assembly v.3.1 (Oar_v3.1) downloaded from the Ensembl database (http://www. ensembl.org/Ovis_aries/Info/Index). Gene expression abundances were estimated with the program Cuffquant from the Cufflinks package (Trapnell et al., 2010) using the Ensembl annotated genes (Oar_v3.1) as a reference. Cuffquant was run using the $-b$ option, which improves the accuracy of transcript abundance estimates by running a new bias detection, and the max-bundle-frags option was adjusted to 3 million of fragments per locus to include highly expressed genes. Gene abundances were normalized by library and gene length with Cuffnorm from Cufflinks (Trapnell et al., 2010) by calculating fragments per kilobase of exon per million fragments mapped (FPKM).

The normalized counts for the target genes included in this study were selected from the Cuffnorm output gene expression file. This file contains a table with the normalized expression levels for each gene in each sample. Once the target genes were selected, the $\mathrm{R}$ software environment (http://www.R-project.org) was used to calculate the mean, the $\mathrm{SD}$, and the coefficient of variation $(\mathbf{C V})$ for each evaluated gene between biological replicates of the same condition. Based on the $\mathrm{CV}$ at each sampling time point, d 10 was selected as the most appropriate day to perform the comparison between both analyzed breeds.

Additionally, a differential expression analysis was performed between the 2 breed groups of milk samples at d 10 of lactation using the Cuffdiff program from Cufflinks (Trapnell et al., 2012). The aim of this analysis was to evaluate whether any of the genes selected as target genes were significantly differentially expressed between the 2 analyzed breeds. Similar to the previously described Cuffquant analysis, Cuffdiff was run using the $-b$ and the -max-bundle-frags options to include highly expressed genes. The CummeRBund Bioconductor $\mathrm{R}$ package was employed to analyze the Cuffdiff outputs. The Venn Diagram function from the limma package in $\mathrm{R}$ (Ritchie et al., 2015) was used to identify the common genes between our list of cheese yield candidate genes and the significant genes highlighted by the differential expression analysis.

\section{RESULTS AND DISCUSSION}

\section{Selection of the Lactation Stage to be Compared: Day 10 of Lactation}

Considering the major objective of our study, which is the comparison of the expression levels of several candidate genes between Churra and Assaf, we performed an initial exploration of the available RNA-Seq data set, which included the transcriptome analysis at d 10, 50, 120, and 150. Normalized FPKM counts and the representation of the gene expression evolution for the 77 target cheese-related genes at the different stages of lactation are shown in supplementary information (Supplementary Tables S1 and S2 and Supplementary Figures S1 to S4; http://dx.doi.org/10.3168/jds.201611048). Based on the average of CV estimates obtained for the 77 considered genes in the 2 breeds across lactation, we found that d 10 was the day showing the lowest average $\mathrm{CV}$ between time point replicates within both breeds (Supplementary Table S3; http://dx.doi. org/10.3168/jds.2016-11048).

In recognition of within-breed variability profiles observed across lactation and to avoid confounding variables such as the length of lactation, the shape of the lactation curve, and so forth, we decided to analyze the first sampling point in both breeds. We considered $d$ 10 as the most suitable time point to perform the comparison between Churra and Assaf dairy sheep breeds, and accordingly, all the subsequent results detailed and discussed in this article are based on the analysis performed on the RNA-Seq data corresponding to d 10 of lactation.

\section{Global Statistics of the RNA-Seq Data}

The RNA sequencing of milk samples at d 10 of lactation produced a total of 264.73 million paired-end reads with an average of 66.18 million paired-end reads per breed. Four biological replicates were analyzed for each breed with paired-end reads ranging from 31.89 to 42.03 million paired-end reads per biological replicate in Assaf sheep and from 23.93 to 36.96 million pairedend reads in Churra sheep. Approximately $86.5 \%$ of the total reads uniquely mapped against the Oar_v3.1 reference genome.

Among all annotated genes in the Oar_v3.1 assembly, the percentage of unique expressed genes at $\mathrm{d} 10$ of lactation was $67.55 \%(17,020)$ in Assaf sheep and $66.67 \%(16,799)$ in Churra sheep, using a threshold of $>0.01$ FPKM to define potentially significant gene expression (Mortazavi et al., 2008). 
The 77 genes selected for this study accounted for approximately $60 \%$ of the total gene FPKM reads, indicating that these genes contributed to a large fraction of the total RNA extracted from MSC. The target genes were classified based on their average gene expression FPKM values into 4 categories: highly expressed genes $(>500$ FPKM), moderately expressed genes $(\geq 10$ FPKM to 500 FPKM), lowly expressed genes $(>0.01$ FPKM to 10 FPKM), and nonexpressed genes $(\leq 0.01$ FPKM). Both breeds had 14 highly expressed genes. The number of genes with moderate expression was similar between Churra and Assaf (41 and 40), and 20 and 18 genes displayed low expression in Churra and Assaf, respectively. Two genes were not expressed in the Churra breed, and 5 were not expressed in the Assaf breed. Among the highly expressed genes in both breeds were the genes codifying caseins and whey proteins (CSN2, CSN3, CSN1S1, ENSOARG00000005099/ PAEP, CSN1S2, LALBA), genes related to lipid metabolism (BTN1A1, XDH, FASN, ADFP, SCD, H$F A B P, A C S S 2)$, and one endogenous protease (CTSB). Among the lowly expressed genes, we observed primarily endogenous proteases (PLAT, SERPINF2, CELA1, and all of the expressed $K L K s), 2$ genes related to lipid metabolism (CEL and SLC27A6), and 2 genes related to citrate metabolism $(A C L Y$ and $P D H X)$. The nonexpressed genes were KLK14 and $P L G$ in the Churra breed and KLK1B,KLK4, KLK8, KLK13, and $P L G$ in the Assaf breed.

\section{Expression Levels of Genes Encoding for Caseins, Whey Proteins, and Lactose-Related Enzymes}

The main proteins in sheep milk are caseins and whey proteins. Caseins constitute the major protein fraction of the milk and comprise 4 fractions: $\alpha_{\mathrm{S}_{1}}$-casein, $\alpha_{\mathrm{S} 2}$-casein, $\beta$-casein, and $\kappa$-casein. These proteins are encoded by CSN1S1, CSN1S2, CSN2, and CSN3, which are genes tightly linked within a 250-kb cluster located on the ovine chromosome 6 (Bevilacqua et al., 2006). Caseins are the most highly expressed genes in sheep MSC (Suárez-Vega et al., 2015); CSN2 is the most highly expressed casein in both the Churra and Assaf breeds at d 10 of lactation (Figure 1a, Supplementary Table S1; http://dx.doi.org/10.3168/jds.2016-11048). This observation is consistent with previous reports on the composition of caseins in which $\beta$-casein constitutes approximately $45 \%$ of the total caseins (Selvaggi et al., 2014). Furthermore, with the exception of $\beta$-casein, caseins were more highly expressed in Churra than in Assaf sheep (Figure 1a). This result agrees with previous reports comparing major milk protein fractions between both breeds, in which Assaf had a lower total casein content than Churra (Rodríguez-Nogales et al.,
2007; Revilla et al., 2009), whereas the results for the $\beta$-casein fraction in these studies were contradictory (Rodríguez-Nogales et al., 2007; Revilla et al., 2009). In sheep milk, large variations in casein concentration exist between individuals and breeds, which greatly influences cheese-making properties (Selvaggi et al., 2014). Variations in casein concentration, mainly in $\beta$-casein and $\kappa$-casein, influence rennet curd formation (Pearse et al., 2009). The main milk whey proteins, $\alpha$-lactalbumin and $\beta$-lactoglobulin, are encoded in sheep by LALBA and PAEP/ENSOARG00000005099, respectively. Moreover, the protein encoded by $L A L B A$, $\alpha$-lactalbumin, stimulates lactose synthesis by interacting with the enzyme of $\beta-1,4$ galactosyl transferase encoded by B4GALT1 (Ebner and Brodbeck, 1968). Therefore, $\alpha$-lactalbumin has a crucial role in milk secretion because it is essential for lactose biosynthesis and for the maintenance of osmotic pressure by stimulating water movement into alveolar lumen (Hayssen and Blackburn, 1985). In this study, although FPKM values for $B 4 G A L T 1$ were similar between the 2 breeds investigated, the expression levels of genes encoding whey proteins were higher in the Assaf breed (Figure 1a, Supplementary Table S1). This observation is supported by higher contents of whey protein fractions having been demonstrated in Assaf compared with Churra sheep (Rodríguez-Nogales et al., 2007; Revilla et al., 2009). The high expression of whey proteins in Assaf could influence the higher milk yield exhibited by this breed compared with Churra sheep.

\section{Expression of Genes Encoding Enzymes Involved in Lipid Metabolism}

Lipid metabolism in the mammary gland is a complex process involving a large number of genes and complex interactions (Bionaz and Loor, 2008). For this analysis, we selected a total of 17 genes encoding proteins that can be grouped under the following lipid metabolism processes: fatty acid (FA) uptake (CEL, LPL, SLC27A6, and $V L D L R$ ), acetate and FA activation and intracellular transport (ACSL1, ACSS2, H-FABP, and $D B I)$, de novo FA synthesis and desaturation ( $A C A C A$, $F A S N$, and $S C D$ ), triacylglyceride (TAG) synthesis (GPAM, LIPIN1, and DGAT1), and milk lipid droplet formation ( $A D F P, X D H$, and BTN1A1).

Among the genes encoding proteins related to FA uptake, the most expressed gene for both breeds at d 10 of lactation was $L P L$ (Figure 1b, Supplementary Table S1; http://dx.doi.org/10.3168/jds.2016-11048). The LPL gene encodes lipoprotein lipase, which anchors very low density lipoprotein or chylomicrons to the mammary endothelium and then hydrolyzes TAG to release FA. The high expression of $L P L$ at d 10 is consistent with 
previous observations reported in cattle, in which $L P L$ was found to be highly expressed at the beginning of lactation (Bionaz and Loor, 2008; Wickramasinghe et al., 2012). The Churra breed exhibited higher expres- sion values than the Assaf breed for both genes involved in active FA uptake ( $L P L$ and $V L D L R$; Figure $1 \mathrm{~b})$.

Fatty acids are esterified with CoA before participating in metabolic pathways (Bionaz and Loor, 2008) and


Figure 1. Bar graph of the FPKM (fragments per kilobase of exon per million fragments mapped) values for the 77 genes selected for this study. The FPKM values are indicated on the $y$-axis, and gene names are indicated on the $x$-axis. Expression values (A) of genes encoding caseins, whey proteins, and lactose synthesis-related enzymes; (B) of genes involved in lipid metabolism; (C) of genes involved in citrate metabolism; and (D) of milk endogenous proteases. 
are then transported to specific organelles (McArthur et al., 1999). Among the genes involved in activation and intracellular channeling, we selected 2 genes related to FA activation, one for the activation of long-chain FA (ACSL1) and one for the activation of short-chain FA (ACSS2), along with 2 genes implicated in FA transport ( $H$-FABP and $D B I$ ). Two of the genes involved in these processes were part of the group of highly expressed genes ( $>500$ FPKM) in the mammary gland for the 2 analyzed breeds, H-FABP and ACSS2 (Figure 1b, Supplementary Table S1; http://dx.doi.org/10.3168/ jds.2016-11048). The more highly expressed gene, $H$ $F A B P$, encodes the FA binding protein, and in addition to its trafficking role, this protein has been shown to prevent the inhibition of $A C A C A$ and $S C D$ (Faergeman et al., 2007), which are genes related to de novo FA synthesis and desaturation. Previous studies in chicken and cow have associated $H$-FABP expression with $S C D$ (Awad et al., 2004; Bionaz and Loor, 2008), suggesting that $\mathrm{H}-\mathrm{FABP}$ provides $\mathrm{FA}$ for $\mathrm{SCD}$, which then releases oleic acid (Bionaz and Loor, 2008). However, in this study, expression levels for $H-F A B P$ were higher in Assaf sheep, and the FPKM values for $S C D$ were higher in Churra sheep (Figure 1b).

The $A C A C A$ and $F A S N$ genes are related to de novo FA synthesis. These genes encode the proteins acetylcoenzyme A carboxylase $\alpha$ and fatty acid synthase, respectively. When examining the FPKM values (Figure 1b, Supplementary Table S1), FASN is a highly expressed gene ( $>500$ FPKM) in both breeds, although its expression is higher in Assaf. The $A C A C A$ gene exhibited lower expression than FASN, similar to what has been reported in dairy cattle (Wickramasinghe et al., 2012).

Stearoyl-CoA desaturase, encoded by $S C D$, is the primary enzyme involved in the synthesis of monounsaturated FA (Ntambi and Miyazaki, 2004). In the ruminant mammary gland, stearoyl-CoA desaturase is responsible for the production of approximately $80 \%$ of the most common form of CLA (cis-9,trans-11 C18:2 isomer), which is secreted in milk (Corl et al., 2001). Compared with cow $[<150$ RPKM in transition lactation (d 15); Wickramasinghe et al., 2012], $S C D$ expression in sheep at d 10 of lactation is high, and the gene is among the highly expressed genes for both breeds (Figure 1b, Supplementary Table S1). The SCD FPKM values were slightly higher in Churra than in Assaf sheep, which could be associated with higher activity of this enzyme in Churra sheep.

Glycerol-3-phosphate O-acyltransferase (GPAM), lipin 1 (LPIN1), and diacylglycerol transferase 1 (DGAT1) encode for enzymes involved in TAG synthesis. Among this group of genes, GPAM had the highest expression (Figure 1b, Supplementary Table S1). These
3 genes exhibited similar expression patterns in Churra and Assaf sheep (Figure 1b).

Adipophilin $(A D F P)$, butyrophilin (BTN1A1), and xanthine dehydrogenase $(X D H)$ are the principal proteins related to milk fat globule formation. This group of genes had the highest expression among the analyzed genes related to fatty acid metabolism (Figure 1b, Supplementary Table S1). This result highlights the importance of lipid droplet formation in the overall scheme of milk lipid metabolism at d 10 of lactation in sheep. $X D H$ was more highly expressed in Assaf sheep, and $A D F P$ and BTN1A1 were slightly more highly expressed in Churra sheep (Figure 1b). The similar expression values of these 3 genes in sheep supports the tripartite model of murine milk lipid secretion (McManaman et al., 2007); however, the high expression of these genes cannot discard the hypothesis of butyrophilin controlling milk fat globule secretion (Robenek et al., 2006).

\section{Expression of Genes Encoding Enzymes Involved in Citrate Metabolism}

Citrate is a normal constituent of milk, and its content directly influences milk-processing properties because it is associated with milk coagulation (Garnsworthy et al., 2006). High levels of citrate in milk are associated with poor coagulation under natural conditions (Sundekilde et al., 2011). Citrate also affects organoleptic characteristics of fermented dairy products because its fermentation products yield distinct aromatic flavors (Rosenthal, 1991). Furthermore, because citrate is an intermediate in the tricarboxylic acid cycle, it plays an indirect role in the de novo synthesis of FA by providing reducing equivalents in the form of NADPH (Faulkner and Peaker, 1982).

To evaluate the expression of genes involved in milk citrate metabolism, we selected 22 genes (Supplementary Table S1; http://dx.doi.org/10.3168/jds.2016-11048) implicated in the tricarboxylic acid cycle (KEGG pathway oas00020) together with some important genes related to citrate content described by Cánovas et al. (2013). Of all genes analyzed, IDH1 (isocitrate dehydrogenase 1) exhibited the highest FPKM values for both breeds (Figure 1c, Supplementary Table S1). Isocitrate dehydrogenase 1 is a cytoplasmic $\mathrm{NADP}(+)$-dependent isocitrate dehydrogenase that plays a significant role in the cytoplasmic production of NADPH to produce citrate (Cánovas et al., 2013). IDH1 is also the isocitrate dehydrogenase that exhibits the highest expression level in Holstein cows (Cánovas et al., 2013), supporting the findings of this study and highlighting the key role of IDH1 in milk citrate content. The expression values of the citrate-related genes we analyzed were similar 
between both breeds. The greatest differences were in the ENSOARG00000012222/PDHB, ACO1, and CS genes, all of which were more highly expressed in the Assaf breed (Figure 1c). Aconitase 1, soluble (ACO1) is a cytosolic protein that catalyzes the conversion of citrate to isocitrate. Pyruvate dehydrogenase (lipoamide) $\beta(P D H B)$ converts pyruvate to acetyl-CoA, providing the primary link between glycolysis and tricarboxylic acid cycle; moreover, citrate synthase $(C S)$ catalyzes the reaction of acetyl-CoA and oxaloacetate to form citrate. The high expression of these 2 related enzymes could lead to a higher citrate content in Assaf milk. To our knowledge, this is the first time that the expression of citrate-related genes has been measured in ovine milk. The citrate content of ovine milk is approximately $47 \%$ less than in bovine milk, with values of 941.4 and 1,768 mg/L, respectively (Holt and Jenness, 1984). Citrate increases milk stability because it has a direct effect on milk pH (Raynal-Ljutovac et al., 2007) and consequently decreases milk coagulation ability. More biochemical assays on milk citrate in sheep are necessary to draw conclusions on how citrate-related enzymes could influence the production of different dairy products.

\section{Expression of Endogenous Proteases}

During cheese ripening, proteolysis contributes to the softening of cheese texture and produces short sapid peptides and amino acids that have a direct influence on flavor (McSweeney, 2004). Hence, the presence of proteases influences the physicochemical characteristics and quality of dairy products. Milk itself is an important source of proteolytic enzymes, and the principal endogenous proteases are plasmin, cathepsins, and elastase (McSweeney, 2004). To evaluate the expression of endogenous proteases in this study, we selected 31 genes (Supplementary Table S1; http://dx.doi.org/10.3168/ jds.2016-11048) encoding cathepsins (CTSA, CTSB, CTSC, CTSF, CTSH, CTSK, CTSL, CTSO, CTSS, $C T S W$, and $C T S Z$ ), elastase 1 (CELA1), and genes related to the plasminogen pathway ( $P L G, P L A T, P L A U$, PLAUR, PLAI-1, and SERPINF2). Additionally, we evaluated the expression of several genes that belong to a family of serine proteases named kallikreins (KLK1, KLK4, KLK5, KLK6, KLK 7, KLK8, KLK10, KLK11, KLK12, KLK13, KLK14, KLK15, and KLKB1). Genes encoding kallikreins were selected based on previous results from our research group that identified some of these genes as being differentially expressed between Churra and Assaf sheep when complete lactation (considering time points d 10,50,120, and 150) was evaluated (Suárez-Vega et al., 2015).
Among the 4 groups of endopeptidases analyzed, genes encoding cathepsins were significantly expressed in the sheep mammary gland (Figure 1d, Supplementary Table S1). Cathepsin B (CTSB) was the most highly expressed gene, with more than 500 FPKM in the Assaf and more than 1,000 FPKM in the Churra breed. Most of the genes encoding cathepsins presented higher expression levels in Churra than in Assaf sheep (Figure 1d).

Plasminogen $(P L G)$ was not expressed in either Churra or Assaf sheep. This result confirms previous studies in dairy cattle that observed that $P L G$ was not expressed in the mammary gland (Berglund et al., 1995; Wickramasinghe et al., 2012) and suggested that milk plasminogen has a sanguineous origin (Kelly and Fox, 2006). However, moderate expression was observed for the remaining genes implicated in the plasminogen pathway, indicating that an active conversion of plasminogen to plasmin occurs in the sheep mammary gland. In general, genes related to plasminogen to plasmin conversion were slightly more highly expressed in Assaf sheep (PLAU and PLAUR), but this result also applies to the plasminogen activator inhibitor (SERPINE1) and the plasmin inhibitor (SERPINF2) (Figure 1d).

Among the remaining analyzed genes, elastase-1 (CELA1) exhibited low expression in milk for both breeds, and the genes encoding kallikreins displayed little or no expression, with the exception of $K L K 10$ and KLK6 in Churra sheep (Figure 1d).

\section{Differentially Expressed Genes}

A total of 181 genes were identified as differentially expressed (q value $<0.05$ ) between Churra and Assaf sheep at D10 of lactation (Supplementary Table S4; http://dx.doi.org/10.3168/jds.2016-11048). Four genes were differentially expressed and had been selected as candidate genes associated with cheese traits for this study: CTSL, CTSK, KLK10, and KLK6, all of which encode for endogenous proteases and are more highly expressed in Churra sheep (Figure 2). These findings confirm previous results that highlight the importance of the expression of genes encoding for kallikreins in Churra sheep milk (Suárez-Vega et al., 2015). Furthermore, we identified 2 other genes encoding endogenous proteases, cathepsins $\mathrm{L}$ and $\mathrm{K}$, which were also differentially expressed in Churra. Hence, the results of the present gene expression analysis suggest that the higher content of endogenous proteases in milk from Churra sheep could be associated with the better aptitude of this breed for the production of mature cheeses compared with Assaf sheep. 


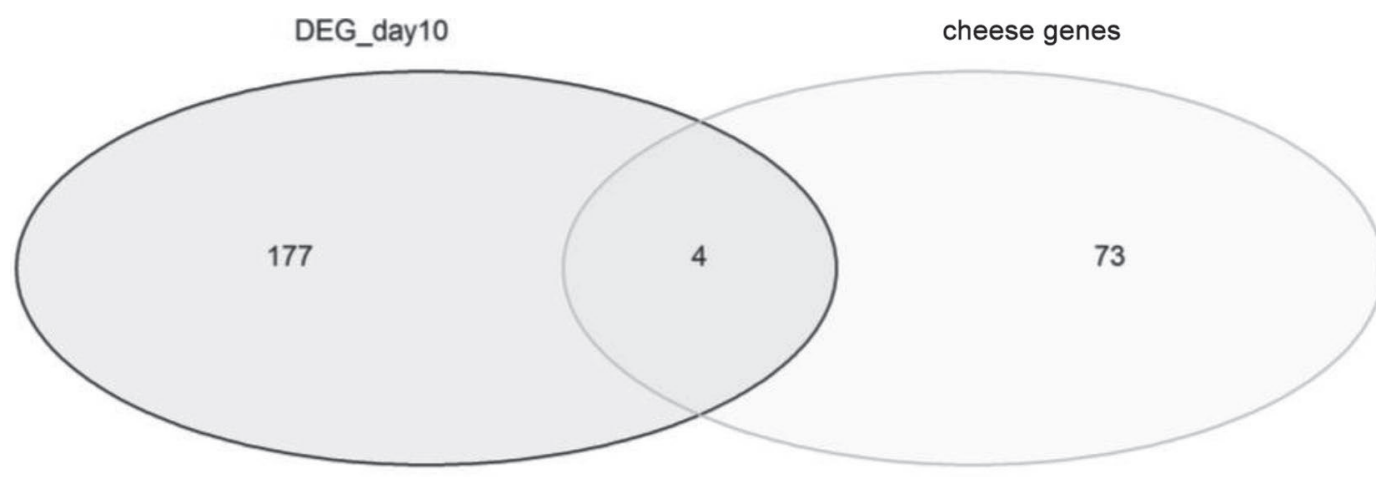

0

Figure 2. Venn diagram illustrating the the 77 candidate genes associated with cheese traits and the genes identified in the differential expression analysis between both breeds (Churra and Assaf). The number of differentially expressed genes (DEG) identified (left ellipse), the number of genes selected for this study (right ellipse), and the genes in common (intersection) are indicated.

Among the 181 differentially expressed genes, we detected 3 other genes that were not included in the list of candidate genes selected for this study but are related to the pathways initially selected with regard to cheese traits: SUCNR1, SERPINE2, and FABP4. SUCNR1, which is more highly expressed in Churra than in Assaf sheep (Figure 3), encodes a succinate receptor, an intermediate molecule of the citric acid cycle. SERPINE2 encodes an inhibitor of serine proteases and was more highly expressed in Assaf sheep (Figure 3 ), suggesting that proteolytic activity is decreased in Assaf milk. FABP 4, which was more highly expressed in Churra sheep (Figure 3), codes for fatty acid binding protein 4 . FABP4 is an intracellular FA transporter with a strong affinity for oleic acid (Bionaz and Loor, 2008). The significantly different expression levels of $F A B P 4$ could be related to the higher content of oleic acid in Churra than in Assaf milk (Lurueña-Martínez et al., 2010a). Additionally, among the differentially expressed genes, we identified 2 genes encoding heat shock proteins, $H_{S P} 70$ and $H S P B 8$, which were up-regulated in Assaf and Churra, respectively, and could influence milk protein synthesis. In general, heat shock proteins constitute a large group of chaperone proteins that
SUCNR1|ENSOARG00000003740

SERPINE2|ENSOARG00000020413

KLK6|ENSOARG00000014290

KLK10|ENSOARG00000014396

HSPB8|ENSOARG00000001872

HSP70|ENSOARG00000008800

FABP4|ENSOARG00000009344

CTSL|ENSOARG00000008683

CTSK|ENSOARG00000020869

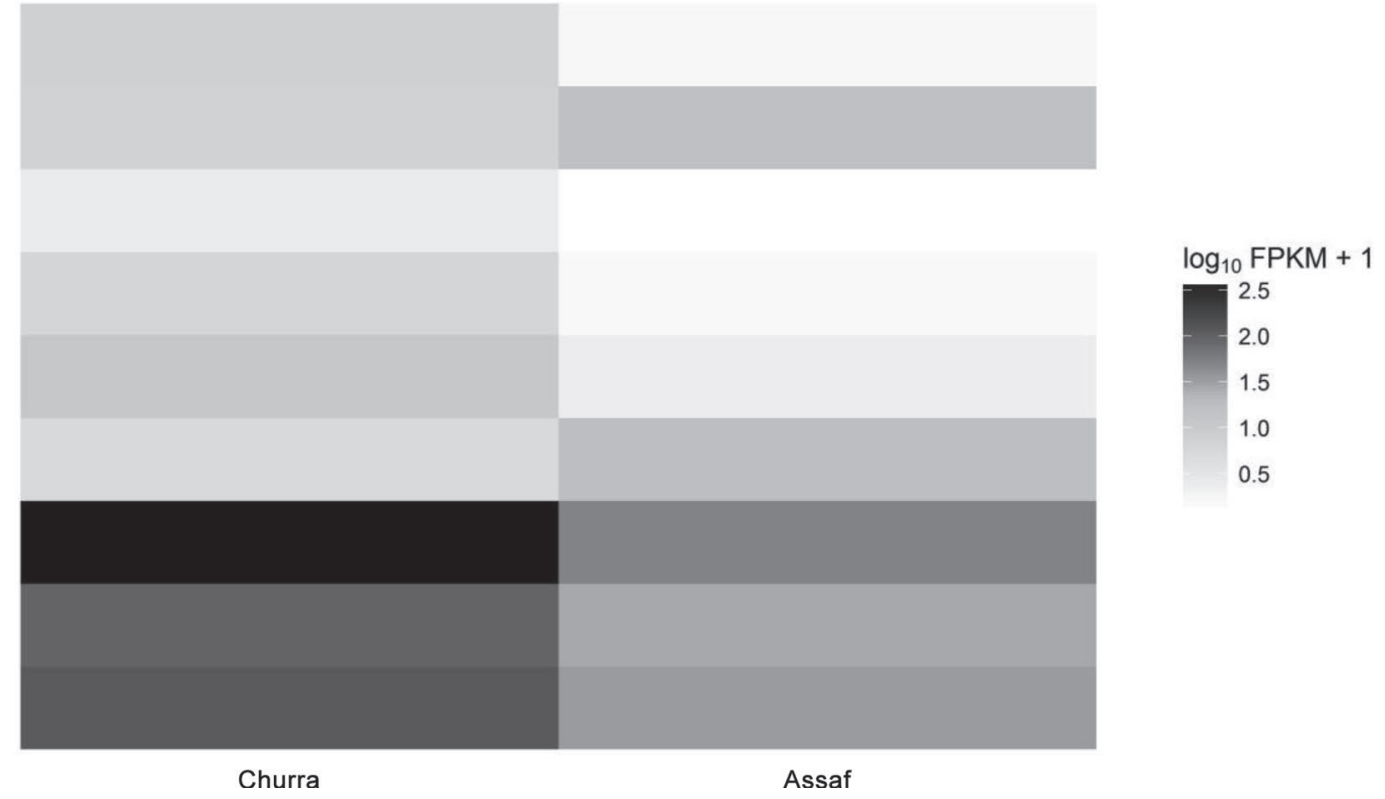

Assaf

Figure 3. Heat map of the differentially expressed genes between Churra and Assaf sheep associated with cheese-related traits. FPKM $=$ fragments per kilobase of exon per million fragments mapped. 
facilitate a wide range of folding processes, including the folding and assembly of newly synthesized proteins, refolding of misfolded and aggregated proteins under stress conditions, membrane translocation of organellar and secretory proteins, and control of the activity of regulatory proteins (Mayer and Bukau, 2005; Treweek et al., 2015).

\section{CONCLUSIONS}

This RNA-Seq study allowed us to analyze the expression levels of 77 genes involved in different important metabolic characteristics (protein, lipids, citrate, and proteases) related to cheese yield and quality traits in sheep. Through this analysis, we identified the genes exhibiting major expression in each of the analyzed pathways that can be considered key genes for traits related to sheep milk cheese. Moreover, the differential expression analysis between Churra and Assaf sheep allowed us to identify 9 genes (CTSL, CTSK, KLK10, KLK6, SUCNR1, SERPINE2, FABP4, HSP70, and $H S P B 8)$ that are significantly differentially expressed between the 2 breeds. The expression levels of these genes could explain part of the differences that the 2 breeds exhibit in relation to milk production and cheese yield and quality traits. Further research is required to confirm these results and to investigate the genetic variation and functional elements underlying the expression differences identified herein.

\section{ACKNOWLEDGMENTS}

This work is included in the framework of the project AGL2012-34437 funded by the Spanish Ministry of Economy and Competitiveness (MINECO, Madrid, Spain). BGG is funded through the Spanish "Ramón y Cajal" Programme (RYC-2012-10230) from the MINECO. The support and availability of the computing facilities of the Foundation of Supercomputing Center of Castile and León (FCSCL, Leon, Spain; http://www. fcsc.es) are also greatly acknowledged.

\section{REFERENCES}

Awad, A. C., H.-S. Shin, D. R. Romsos, and J. I. Gray. 2004. Direct desaturation of free myristic acid by hen liver microsomal Delta9desaturase without prior activation to myristoyl-CoA derivative. J. Agric. Food Chem. 52:3194-3201. http://dx.doi.org/10.1021/ jf0348020.

Berglund, L., M. D. Andersen, and T. E. Petersen. 1995. Cloning and characterization of the bovine plasminogen cDNA. Int. Dairy J. 5:593-603. http://dx.doi.org/10.1016/0958-6946(94)00033-L.

Bevilacqua, C., J. C. Helbling, G. Miranda, and P. Martin. 2006. Translational efficiency of casein transcripts in the mammary tissue of lactating ruminants. Reprod. Nutr. Dev. 46:567-578. http:// dx.doi.org/10.1051/rnd:2006028.
Bionaz, M., and J. J. Loor. 2008. Gene networks driving bovine milk fat synthesis during the lactation cycle. BMC Genomics 9:366 http://dx.doi.org/10.1186/1471-2164-9-366.

Cánovas, A., G. Rincon, C. Bevilacqua, A. Islas-Trejo, P. Brenaut, R. C. Hovey, M. Boutinaud, C. Morgenthaler, M. K. VanKlompenberg, P. Martin, and J. F. Medrano. 2014. Comparison of five different RNA sources to examine the lactating bovine mammary gland transcriptome using RNA-sequencing. Sci. Rep. 4:5297 http://dx.doi.org/10.1038/srep05297.

Cánovas, A., G. Rincón, A. Islas-Trejo, R. Jimenez-Flores, A. Laubscher, and J. F. Medrano. 2013. RNA sequencing to study gene expression and single nucleotide polymorphism variation associated with citrate content in cow milk. J. Dairy Sci. 96:2637-2648. http://dx.doi.org/10.3168/jds.2012-6213.

Corl, B. A., L. H. Baumgard, D. A. Dwyer, J. M. Griinari, B. S. Phillips, and D. E. Bauman. 2001. The role of Delta(9)-desaturase in the production of cis-9, trans-11 CLA. J. Nutr. Biochem. $12: 622-630$

Dobin, A., C. A. Davis, F. Schlesinger, J. Drenkow, C. Zaleski, S. Jha, P. Batut, M. Chaisson, and T. R. Gingeras. 2013. STAR: Ultrafast universal RNA-seq aligner. Bioinformatics 29:15-21. http:// dx.doi.org/10.1093/bioinformatics/bts635.

Ebner, K., and U. Brodbeck. 1968. Biological role of alpha-lactalbumin: A review. J. Dairy Sci. 51:317-322.

Faergeman, N. J., M. Wadum, S. Feddersen, M. Burton, B. B. Kragelund, and J. Knudsen. 2007. Acyl-CoA binding proteins; structural and functional conservation over 2000 MYA. Mol. Cell. Biochem. 299:55-65. http://dx.doi.org/10.1007/s11010-005-9040-3.

Faulkner, A., and M. Peaker. 1982. Reviews of the progress of dairy science: Secretion of citrate into milk. J. Dairy Res. 49:159-169.

Garnsworthy, P. C., L. L. Masson, A. L. Lock, and T. T. Mottram. 2006. Variation of milk citrate with stage of lactation and de novo fatty acid synthesis in dairy cows. J. Dairy Sci. 89:1604-1612. http://dx.doi.org/10.3168/jds.S0022-0302(06)72227-5.

Gonzalo, C., J.A. Carriedo, J.D. Gomez, L.D. Gomez, and F. San Primitivo. 1994. Diurnal variation in the somatic cell count of ewe milk. J. Dairy Sci. 77:1856-1859. http://dx.doi.org/S00220302(94) $77127-7$.

Hayssen, V., and D. G. Blackburn. 1985. $\alpha$-Lactalbumin and the origins of lactation. Evolution (N. Y.) 39:1147-1149.

Holt, C., and R. Jenness. 1984. Interrelationships of constituents and partition of salts in milk samples from eight species. Comp. Biochem. Physiol. A Comp. Physiol. 77:275-282.

Kelly, A. L., and P. F. Fox. 2006. Indigenous enzymes in milk: A synopsis of future research requirements. Int. Dairy J. 16:707-715. http://dx.doi.org/10.1016/j.idairyj.2005.10.018.

Lurueña-Martínez, M. A., C. Palacios, A. M. Vivar-Quintana, and I. Revilla. 2010a. Effect of the addition of calcium soap to ewes' diet on fatty acid composition of ewe milk and subcutaneous fat of suckling lambs reared on ewe milk. Meat Sci. 84:677-683. http:// dx.doi.org/10.1016/j.meatsci.2009.11.002.

Lurueña-Martinez, M. A., I. Revilla, P. Severiano-Perez, and A. M. Vivar-Quintana. 2010b. The influence of breed on the organoleptic characteristics of Zamorano sheep's raw milk cheese and its assessment by instrumental analysis. Int. J. Dairy Technol. 63:216-223. http://dx.doi.org/10.1111/j.1471-0307.2010.00565.x.

Mayer, M. P., and B. Bukau. 2005. Hsp70 chaperones: Cellular functions and molecular mechanism. Cell. Mol. Life Sci. 62:670-684. http://dx.doi.org/10.1007/s00018-004-4464-6.

McArthur, M. J., B. P. Atshaves, A. Frolov, W. D. Foxworth, A. B. Kier, and F. Schroeder. 1999. Cellular uptake and intracellular trafficking of long chain fatty acids. J. Lipid Res. 40:1371-1383.

McManaman, J. L., T. D. Russell, J. Schaack, D. J. Orlicky, and H. Robenek. 2007. Molecular determinants of milk lipid secretion. J. Mammary Gland Biol. Neoplasia 12:259-268. http://dx.doi. org/10.1007/s10911-007-9053-5.

McSweeney, P. L. H. 2004. Biochemistry of cheese ripening. Int. J. Dairy Technol. 57:127-144. http://dx.doi.org/10.1111/j.14710307.2004.00147.x. 
Moioli, B., M. D'Andrea, and F. Pilla. 2007. Candidate genes affecting sheep and goat milk quality. Small Rumin. Res. 68:179-192. http://dx.doi.org/10.1016/j.smallrumres.2006.09.008.

Mortazavi, A., B. A. Williams, K. McCue, L. Schaeffer, and B. Wold, 2008. Mapping and quantifying mammalian transcriptomes by RNA-Seq. Nat. Methods 5:621-628. http://dx.doi.org/10.1038/ nmeth.1226.

Ntambi, J. M., and M. Miyazaki. 2004. Regulation of stearoyl-CoA desaturases and role in metabolism. Prog. Lipid Res. 43:91-104.

Othmane, M. H., L. F. De La Fuente, J. A. Carriedo, and F. San Primitivo. 2002. Heritability and genetic correlations of test day milk yield and composition, individual laboratory cheese yield, and somatic cell count for dairy ewes. J. Dairy Sci. 85:2692-2698. http://dx.doi.org/10.3168/jds.S0022-0302(02)74355-5.

Pearse, M. J., P. M. Linklater, R. J. Hall, and A. G. Mackinlay. 2009 Effect of casein micelle composition and casein dephosphorylation on coagulation and syneresis. J. Dairy Res. 53:381-390. http:// dx.doi.org/10.1017/S0022029900025000.

Pérez-Cabal, M. Á., E. Legaz, I. Cervantes, L. F. De La Fuente, R. Martínez, F. Goyache, and J. P. Gutiérrez. 2013. Association between body and udder morphological traits and dairy performance in Spanish Assaf sheep. Arch. Tierzucht 56:430-442. http://dx.doi. org/10.7482/0003-9438-56-042.

Peris, C., P. Molina, N. Fernandez, M. Rodriguez, and A. Torres. 1991. Variation in somatic cell count, California mastitis test, and electrical conductivity among various fractions of ewe's milk. J. Dairy Sci. 74:1553-1560. http://dx.doi.org/10.3168/jds.S00220302(91)78317-3.

Raynal-Ljutovac, K., Y. W. Park, F. Gaucheron, and S. Bouhallab. 2007. Heat stability and enzymatic modifications of goat and sheep milk. Small Rumin. Res. 68:207-220.

Revilla, I., J. M. Rodríguez-Nogales, and A. M. Vivar-Quintana 2009. Effect of somatic cell counts on ewes' milk protein profile and cheese-making properties in different sheep breeds reared in Spain. J. Dairy Res. 76:210-215. http://dx.doi.org/10.1017/ S0022029909004002.

Ritchie, M. E., B. Phipson, D. Wu, Y. Hu, C. W. Law, W. Shi, and G. K. Smyth. 2015. limma powers differential expression analyses for RNA-sequencing and microarray studies. Nucleic Acids Res. 43:e47 http://dx.doi.org/10.1093/nar/gkv007.

Robenek, H., O. Hofnagel, I. Buers, S. Lorkowski, M. Schnoor, M. J. Robenek, H. Heid, D. Troyer, and N. J. Severs. 2006. Butyrophilin controls milk fat globule secretion. Proc. Natl. Acad. Sci. USA 103:10385-10390. http://dx.doi.org/10.1073/pnas.0600795103.

Rodríguez-Nogales, J. M., A. M. Vivar-Quintana, and I. Revilla. 2007. Influence of somatic cell count and breed on capillary electrophoretic protein profiles of ewes' milk: A chemometric study. J. Dairy Sci. 90:3187-3196. http://dx.doi.org/10.3168/jds.2006-589.

Rosenthal, I. 1991. Milk and Dairy Products. Properties and Processing. VCH Publishers, New York, NY.

Selvaggi, M., V. Laudadio, C. Dario, and V. Tufarelli. 2014. Investigating the genetic polymorphism of sheep milk proteins: A useful tool for dairy production. J. Sci. Food Agric. 94:3090-3099. http:// dx.doi.org/10.1002/jsfa.6750.

Suárez-Vega, A., B. Gutiérrez-Gil, C. Klopp, C. Robert-Granie, G. Tosser-Klopp, and J. J. Arranz. 2015. Characterization and comparative analysis of the milk transcriptome in two dairy sheep breeds using RNA sequencing. Sci. Rep. 5:18399 http://dx.doi. org/10.1038/srep18399.

Sundekilde, U. K., P. D. Frederiksen, M. R. Clausen, L. B. Larsen, and H. C. Bertram. 2011. Relationship between the metabolite profile and technological properties of bovine milk from two dairy breeds elucidated by NMR-based metabolomics. J. Agric. Food Chem. 59:7360-7367. http://dx.doi.org/10.1021/jf202057x.

Trapnell, C., A. Roberts, L. Goff, G. Pertea, D. Kim, D. R. Kelley, H. Pimentel, S. L. Salzberg, J. L. Rinn, and L. Pachter. 2012. Differential gene and transcript expression analysis of RNA-seq experiments with TopHat and Cufflinks. Nat. Protoc. 7:562-578. http://dx.doi.org/10.1038/nprot.2012.016.

Trapnell, C., B. A. Williams, G. Pertea, A. Mortazavi, G. Kwan, M. J. van Baren, S. L. Salzberg, B. J. Wold, and L. Pachter. 2010. Transcript assembly and quantification by RNA-Seq reveals unannotated transcripts and isoform switching during cell differentiation. Nat. Biotechnol. 28:511-515. http://dx.doi.org/10.1038/nbt.1621.

Treweek, T. M., S. Meehan, H. Ecroyd, and J. A. Carver. 2015 Small heat-shock proteins: important players in regulating cellular proteostasis. Cell. Mol. Life Sci. 72:429-451. http://dx.doi org/10.1007/s00018-014-1754-5.

Wang, Z., M. Gerstein, and M. Snyder. 2009. RNA-Seq: A revolutionary tool for transcriptomics. Nat. Rev. Genet. 10:57-63.

Wickramasinghe, S., G. Rincon, A. Islas-Trejo, and J. F. Medrano. 2012. Transcriptional profiling of bovine milk using RNA sequencing. BMC Genomics 13:45 http://dx.doi.org/10.1186/1471-2164$13-45$. 